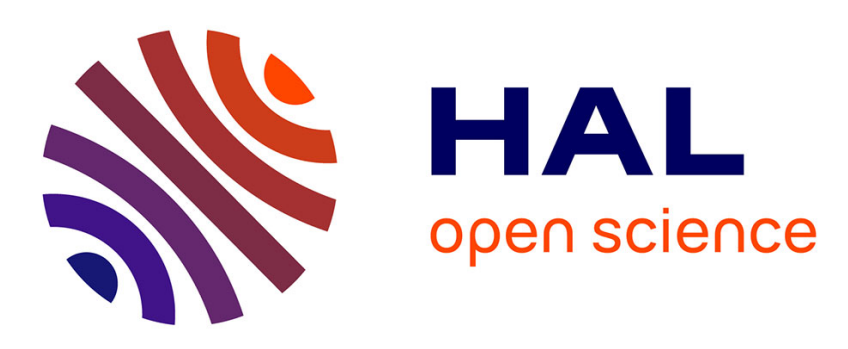

\title{
Profils sociodémographique et sportif des usagers des snowparks dans les stations de montagne des Alpes françaises
}

\author{
Véronique Reynier, Bastien Soulé, Johanne Pabion-Mouriès
}

\section{To cite this version:}

Véronique Reynier, Bastien Soulé, Johanne Pabion-Mouriès. Profils sociodémographique et sportif des usagers des snowparks dans les stations de montagne des Alpes françaises. Leisure/Loisir, 2018, 42 (2), pp.149-162. 10.1080/14927713.2018.1449132 . hal-02126661

\section{HAL Id: hal-02126661 \\ https: / hal.univ-grenoble-alpes.fr/hal-02126661}

Submitted on 4 Jun 2021

HAL is a multi-disciplinary open access archive for the deposit and dissemination of scientific research documents, whether they are published or not. The documents may come from teaching and research institutions in France or abroad, or from public or private research centers.
L'archive ouverte pluridisciplinaire HAL, est destinée au dépôt et à la diffusion de documents scientifiques de niveau recherche, publiés ou non, émanant des établissements d'enseignement et de recherche français ou étrangers, des laboratoires publics ou privés. 
Reynier, V., Soulé, B., \& Pabion-Mouriès, J. (2018). Profils

sociodémographique et sportif des usagers des snowparks dans les stations

de montagne des Alpes françaises. Leisure/Loisir, 42(2), 149-162.

https://doi.org/10.1080/14927713.2018.1449132

\section{Introduction}

Depuis les années 2000, les snowparks ${ }^{1}$ contribuent fortement à l'attractivité touristique et au positionnement des stations de montagne (Guibert, 2006 ; Soulé, Reynier, \& Pabion-Mouriès, 2015). De plus en plus nombreuses ${ }^{2}$ et massivement fréquentées, ces pistes spécifiques aménagées sont devenues des lieux d'évolution acrobatique pour de nombreux pratiquants des sports d'hiver, que ce soit de manière occasionnelle ou assidue. Certains clients des stations fréquentent exclusivement ces espaces, délaissant les pistes traditionnelles.

Pourtant, on en sait relativement peu sur les différents profils d'usagers des snowparks. A ce jour, la littérature scientifique s'est en effet focalisée sur deux aspects : primo, les modes de vie et codes culturels propres à la communauté des adeptes de freestyle ${ }^{3}$, intégrant la rivalité originelle entre pratiquants du surf des neiges et skieurs, ainsi que la domination masculine qui s'opère en snowpark (Anderson, 1999 ; Coates, Clayton, \& Humberstone, 2010 ; Edensor \& Richards, 2007 ; Heino, 2000 ; Thorpe, 2004, 2012) ; secundo, l'épidémiologie des usagers des snowparks, qui révèle leur nette surexposition au risque de blessures graves et d'hospitalisation (Brooks, Evans, \& Rivara, 2010 ; Goulet, Hagel, Hamel, \& Légaré, 2007 ; Laporte, 2011 ; Ruedl, Kopp, Sommersacher, Woldrich, \& Burtscher, 2013 ; Russell et al.,

\footnotetext{
${ }^{1} \mathrm{Si}$ au Canada, le terme snowparks peut être traduit par «parcs à neige », la correspondance en France n'est pas appropriée. En effet, dans les stations de montagne françaises, les « parcs à neige » désignent avant tout des espaces dévolus à l'initiation au ski des très jeunes enfants (3 à 6 ans). Le terme snowpark est communément adopté pour décrire, en station, les espaces circonscrits à la pratique acrobatique.

${ }^{2}$ De 10 snowparks dans les stations de montagne françaises, dans les années 1990 (Curtet, 2007), on est passé à une vingtaine dans les années 2000, puis à plus de 50 en 2010 (Curtet, 2011; Feuillie, 2011).

${ }^{3}$ Le freestyle (ou «style libre») est une discipline olympique; le terme usuel correspond toutefois plus largement à un style de pratique « libre », en opposition à la pratique traditionnelle du ski de descente.
} 
2013 ; Torjussen \& Bahr, 2006). D'autres dimensions sont plus ponctuellement investiguées, comme la nature de l'engagement corporel des adeptes du freestyle (Pabion-Mouriès, Reynier, \& Soulé, 2014) ou leur usage intensif des NTIC (vidéo, Internet et réseaux sociaux) (Woermann, 2012).

Essentiellement focalisés sur le noyau dur des insiders ${ }^{4}$, ces travaux, dont les dispositifs méthodologiques privilégient une orientation qualitative, délaissent quelque peu la pluralité des profils d'usagers de ces espaces récréatifs. En France, cette diversité est d'autant plus marquée que contrairement à ce qui se passe, par exemple, aux Etats-Unis (Thorpe, 2011), un simple titre de transport permet d'accéder à l'ensemble du domaine skiable d'une station, snowpark compris.

Nous présentons dans cet article les résultats issus d'un questionnaire adressé durant l'hiver 2014, en face-à-face et in situ, à 918 pratiquants des sports d'hiver en snowpark dans les Alpes françaises. La variété des profils d'usagers, des modalités de pratique et motivations mises en avant, jusqu'alors peu abordée dans les études antérieures, est notamment soulignée.

\section{Contexte : l'avènement du freestyle en snowpark}

En station de montagne, le freestyle puise ses origines dans l'évolution de la pratique du surf des neiges, apparue en France dans les années 1970 sur fond de recherche de sensations, d'hédonisme et de nouveauté. Pratique sportive alternative et distinctive (Rinehart \& Sydnor, 2003), le surf des neiges s'oppose alors à la pratique du ski alpin, considérée comme rigide, cadrée et excluant toute créativité (Reynier \& Chantelat, 2005 ; Wheaton \& Beal, 2003). Dans les années 1990, le surf des neiges est consacré aux yeux du public comme l'étendard de la contre-culture au sein des stations de sports d'hiver. Il va néanmoins être peu à peu rattrapé par un processus de massification, de «sportivisation » et de commercialisation (Humphreys, 1997 ; Heino, 2000).

En opposition à cette normalisation du surf des neiges, le freestyle s'est développé à partir de la fin des années 1980, en référence également à la culture urbaine, notamment par

\footnotetext{
${ }^{4}$ La notion d'insider détermine, dans ce contexte, les membres de la subculture du freestyle. Elle insiste sur l'appartenance à la communauté des freestylers (partage de codes distinctifs, adhésion à des valeurs communes, etc.) et s'oppose à la notion d'outsider correspondant aux membres extérieurs à cette communauté.
} 
l'entremise d'anciens pratiquants de la planche à roulettes, communément appelée en anglais «skateboard». Par un transfert des habitudes de pratiques, cette communauté privilégie la réalisation de figures acrobatiques jugées sur leur difficulté technique, leur enchaînement, le risque associé et leur côté spectaculaire (Vermeir \& Reynier, 2008). Ces performances se distinguent également de la pratique traditionnelle des sports d'hiver par l'unité de lieu : le snowpark est un site confiné à un espace relativement restreint, généralement équivalent à une section de piste tout au plus. Initialement réservé aux adeptes du surf des neiges, le freestyle a par la suite fait de nombreux émules dans le monde du ski (Drouet \& Kemo Keimbou, 2005 ; Dupuy, 2007; Apilli, 2007), sur fond de renouveau de cette pratique (Curtet, 2007), mais aussi de stagnation puis de régression de la pratique du surf des neiges (Lamprecht et al. 2015a), particulièrement chez les adolescents (Lamprecht et al. 2015b) ${ }^{5}$.

\section{Méthodologie}

Les résultats présentés dans cet article sont issus d'une étude plus large, financée par la Fondation MAIF (Mutuelle d'Assurance des Instituteurs de France), portant sur l'accidentologie et le rapport au risque des usagers des snowpark. Les connaissances de base relatives aux profils sociodémographique et sportif des adeptes des snowparks s'avérant lacunaires, nous avons inclus un certain nombre de questions relatives à ces dimensions. Elles ont au final revêtu une part d'autant plus importante que nous avions besoin, dans une perspective accidentologique, d'informations précises quant aux différentes catégories de populations fréquentant ces espaces. Dans le cadre de cet article, seuls ces résultats seront détaillés. Ils concernent 1) la caractérisation de la pratique en snowpark: type de pratique (ski, surf des neiges ou autre), fréquence, niveau, modalités de pratique (type de modules préférentiellement empruntés) ; 2) les caractéristiques sociodémographiques des pratiquants (âge, sexe, etc.) ; 3) les raisons incitant à fréquenter les snowparks (en parallèle ou en remplacement des pistes traditionnelles.

Les modalités de réponse proposées pour chacune des questions destinées à caractériser et mesurer ces différentes dimensions ont été construites à partir d'une pré-enquête qualitative. Celle-ci a consisté en la réalisation de 32 entretiens semi-directifs auprès de personnes

\footnotetext{
${ }^{5}$ Les enquêtes sur lesquelles se base le constat d'un déclin de la pratique du surf des neiges en station de montagne ont été réalisées en Suisse. En l'absence d'étude sociodémographique fiable relative à la situation sur le territoire français, nous émettons l'hypothèse d'une similarité des tendances entre la Suisse et la France.
} 
fréquentant les snowparks et présentant des caractéristiques sociodémographiques et sportives diversifiées (fréquence, niveau et modalité de pratique). Le questionnaire définitif a été élaboré à la suite de deux pré-tests ayant pour objectif d'évaluer l'intelligibilité du questionnaire, de repérer et de corriger certaines de ses imperfections.

Bien que la détermination a priori de la taille d'un échantillon laisse beaucoup de place à l'empirisme (Berthier \& Berthier, 1971), nous avons choisi d'interroger un millier de personnes, ce nombre semblant suffisant pour obtenir des résultats d'une précision acceptable, notamment en ce qui concerne les sous-groupes. Afin d'obtenir un échantillon représentant au mieux la population des personnes fréquentant les snowparks, nous nous sommes rendus dans différents types de stations (12 au total se répartissant sur les départements d'Isère, de Savoie, de Haute-Savoie et des Hautes Alpes). Les 20 journées consacrées à la passation des questionnaires ont été choisies de telle sorte qu'elles couvrent les périodes de vacances scolaires des différentes zones, les périodes hors vacances, les week-ends et les jours de la semaine. Les enfants de moins de dix ans n'ont pas été interrogés, le sens de certaines questions pouvant être difficilement compréhensible pour eux.

Dans les snowparks, nous cherchions à interroger l'ensemble des personnes présentes, et ce quelles que soient leurs modalités de pratique. Les questionnaires ont tous été remplis en faceà-face. En fonction des préférences de l'enquêté, soit l'enquêteur lisait les questions et inscrivait ses réponses, soit l'enquêté remplissait seul le questionnaire et le remettait à l'enquêteur une fois complété. La passation, qui s'est effectuée de fin janvier à début avril 2014, a finalement permis de recueillir 918 questionnaires exploitables.

La significativité des différences annoncées dans les résultats a systématiquement été vérifiée, le seuil de significativité choisi étant de 0,05. Les comparaisons de moyennes ont été testées à l'aide des tests de Student et/ou de Fischer; les comparaisons de pourcentages avec celui du Khi-deux.

\section{Résultats}

Les résultats présentés dans cette section de l'article sont tirés de l'enquête quantitative par questionnaire. 


\section{Spécificités de la fréquentation des snowparks}

Trente pour cent des personnes se rendant dans les snowparks n'empruntent pas les différents modules, se contentant de les contourner ou de les longer; nous les nommerons les visiteurs. Les femmes sont davantage représentées dans cette catégorie, même si elles restent minoritaires (38,2\%). La moyenne d'âge des visiteurs est plus élevée que celle des pratiquants (25 ans contre 20 ans). Il est intéressant de noter que si 37,5\% des visiteurs se rendent exceptionnellement dans les snowparks, la majorité (50,9\%) s'y retrouve au moins de temps en temps ; il s'agit même d'une occupation principale pour $11,7 \%$ d'entre eux.

Les autres, désignés par le terme de pratiquants, font essentiellement des sauts $(50,7 \%$ d'entre eux), ou des rails et des sauts dans des proportions similaires $(41,9 \%)$; ils sont très peu nombreux $(7,4 \%)$ à privilégier la pratique des rails. L'ensemble des personnes présentes dans les snowparks (visiteurs et pratiquants) seront appelés usagers.

Pour 54,2\% des pratiquants, il s'agit d'une activité à part entière : ils passent généralement l'essentiel de leur journée en snowpark (20,1\% des pratiquants ne fréquentent que ces espaces et $34,2 \%$ y restent principalement). Remarquons néanmoins la large part $(39,7 \%)$ prise par ceux qui se rendent seulement de temps en temps, lors de leur journée en station, dans les snowparks, et la très petite minorité $(6,1 \%)$ que représentent ceux qui s'y rendent exceptionnellement.

\section{Caractéristiques sociodémographiques}

$81,1 \%$ des usagers des snowparks sont des hommes, proportion qui monte à $89,8 \%$ si l'on se focalise sur les pratiquants. Cette faible proportion de femmes dans les snowparks contraste avec ce qui se passe sur le reste du domaine skiable, où les hommes et les femmes sont représentés dans des proportions similaires (Vermeir \& Reynier, 2008). 62,2\% des femmes fréquentant les snowparks sont des visiteuses n'empruntant pas les différents modules.

La moyenne d'âge des pratiquants dans les snowparks est de 20 ans (avec un écart type de 7,3 ans); la médiane est de 18 ans. La classe d'âge la plus représentée est celle des 15-18 ans, qui représente $30,7 \%$ des pratiquants; suivent les $19-25$ ans (26,9\%) puis les plus de 25 ans $(21,4 \%)$ et les enfants âgés de 10 à 14 ans $(21 \%)$. Lorsque l'on prend en compte l'ensemble 
des personnes présentes sur ces espaces aménagés, la moyenne d'âge et l'écart-type s'accroissent (respectivement 21,5 ans et 9,6 ans) ; cet effet provient de la forte proportion des plus de 35 ans parmi les visiteurs.

\section{Modalités et niveau de pratique}

Si les skieurs sont largement majoritaires $(63,1 \%)$ parmi les pratiquants dans les snowparks, la sur-représentation des pratiquants de surf des neiges est à souligner ; ces derniers représentent en effet plus du tiers des pratiquants $(34,8 \%)$, alors que leur proportion sur les pistes serait moindre (elle est évaluée à 17\%) (Association des Médecins de Montagne, 2013).

Le niveau «moyen » est le plus représenté en snowpark $(44,2 \%)$ et deux pratiquants sur dix sont des débutants $(21,6 \%)^{6}$. Les experts, à même de réaliser des sauts comportant plusieurs rotations sur plusieurs axes, représentent $13,8 \%$ des pratiquants et les confirmés 20,4\%. Malgré ce niveau de pratique relativement peu élevé, $79 \%$ des pratiquants se rendent sur des modules difficiles. 54,4\% des débutants empruntent des modules de difficulté moyenne, alors que $59,5 \%$ des pratiquants de niveau moyens vont sur des modules difficiles. La plupart des confirmés $(59,5 \%)$ et des experts $(91,7 \%)$ se confrontent quant à eux aux modules d'un niveau très difficile.

\section{Insérer ici figure 1}

\footnotetext{
${ }^{6}$ Ces niveaux correspondent à ceux déclarés par les pratiquants, sur la base d'une définition précise de chacun d'eux : débutant (saut droit de petite amplitude, rail relativement large) ; moyen (saut d'une rotation ou saut droit de grande amplitude) ; confirmé (saut avec plusieurs rotations) ; expert (plusieurs rotations sur plusieurs axes).
} 
Les pratiquantes et les pratiquants ne diffèrent pas significativement selon leur âge, leur type de pratique (ski ou surf des neiges), la fréquence avec laquelle ils se rendent en station ainsi que leurs modalités de pratique (rails, sauts etc.). En revanche, le niveau de pratique en freestyle se trouve être une variable fortement différenciatrice entre hommes et femmes : deux fois plus de femmes que d'hommes (41,3\% contre 19,4\%) se déclarent d'un niveau débutant et trois fois moins $(5,8 \%$ contre $14,8 \%)$ d'un niveau expert.

Au cours d'une journée de ski, la fréquentation du snowpark par les femmes est sensiblement différente de celle des hommes. Tandis que 55,6\% des pratiquants fréquentent uniquement ou essentiellement les snowparks, $56,9 \%$ des pratiquantes s'y rendent « de temps en temps » ou « exceptionnellement ».

Variation de la pratique et de la fréquentation selon l'âge

Une différence d'importance entre les pratiquants de surf des neiges et de ski est leur âge. En effet, la moyenne d'âge des pratiquants du surf des neiges est de 22,7 ans contre 18,4 ans pour les skieurs. Chez les enfants âgés de 10 à 14 ans, les pratiquants du surf des neiges sont largement minoritaires : ils représentent $19,7 \%$ des pratiquants des snowparks de cette tranche d'âge. Cette proportion ne cesse de croitre au fur et à mesure que les classes d'âge augmentent, pour atteindre $63,2 \%$ chez les plus de 25 ans.

Si le lien existant entre les classes d'âge et le niveau de pratique montre que ce dernier augmente au fur et à mesure de l'avancée en âge, le niveau élevé de pratique des enfants, comparativement au niveau global, est à souligner : près du tiers des 10-14 ans ont déjà un niveau leur permettant d'effectuer des sauts avec plusieurs rotations (confirmé et expert).

\section{Insérer ici figures 2 et 3}

Variation des modalités de pratique et de fréquentation selon le type de pratique

Parmi l'ensemble des usagers, seuls 14,3\% de ceux qui font du surf des neiges n'utilisent pas les différents modules (proportion qui s'élève à 35,9\% chez les skieurs). Les adeptes du surf 
des neiges se distinguent également par leur plus forte proportion d'hommes $(87,5 \%$ contre $79,2 \%$ chez les skieurs). En revanche, lorsque l'on s'intéresse uniquement aux pratiquants, les hommes et les femmes sont représentés en surf des neiges et en ski dans des proportions équivalentes (10,9\% pour le premier et 9,5\% pour le second). Au cours de leur journée en station, les usagers qui font du surf des neiges sont également plus nombreux à fréquenter assidument les snowparks (la moitié d'entre eux y passent l'essentiel, voire la totalité de leur temps), mais cette différence ne perdure pas lorsque l'on s'intéresse uniquement aux pratiquants. Le niveau de pratique déclaré par les pratiquants de surf des neiges et les skieurs est identique, et il en va de même en ce qui concerne leur utilisation des différents types de modules (modules à plat ou modules de saut). Par contre, une différence apparaît concernant le niveau de difficulté des modules qu'ils empruntent : les skieurs sont bien plus nombreux que les pratiquants de surf des neiges à emprunter des modules de niveau très difficile (noir) $(49,3 \%$ contre $26,5 \%)$.

La prise en compte du niveau des pratiquants dans cette relation montre que les skieurs et les pratiquants de surf des neiges débutants se comportent de manière similaire en termes de niveau des modules empruntés ; puis, dès qu'ils atteignent un niveau leur permettant d'effectuer des sauts d'une rotation (niveau moyen), les skieurs s'affrontent à des modules plus difficiles que ne le font les pratiquants de surf des neiges. Cette tendance se vérifie aussi pour les pratiquants confirmés et experts.

\section{Insérer ici figures 4 et 5}

\section{Les raisons de la pratique en snowpark}

Les raisons poussant les individus à fréquenter les snowparks ont été mesurées à partir des réponses obtenues à la question fermée suivante : «quelles sont les principales raisons pour lesquelles tu te rends dans un snowpark (plusieurs choix possibles)? ». Les modalités de réponses ont été proposées à partir d'une analyse thématique des entretiens effectués lors de la phase qualitative de la recherche (cf. méthodologie).

Les deux principales raisons poussant les pratiquants à se rendre dans les snowparks sont la pratique du freestyle (61\%) et la recherche de sensations (58\%); suivent l'aspect ludique de la 
pratique (48\%) et l'ambiance des lieux (46\%). Les visiteurs sont, quant à eux, principalement motivés par le fait d'aller regarder ceux qui pratiquent.

\section{Insérer ici figure 6}

Ces raisons diffèrent selon les niveaux de pratique. Ainsi, la pratique du freestyle motive plus de $80 \%$ des experts et des confirmés, contre seulement $29 \%$ des débutants ; chez ces derniers, ce sont davantage le désir de «skier autrement» et «d'essayer de nouvelles choses » qui les poussent à fréquenter les snowparks. Les débutants sont également quatre fois plus nombreux que les experts à dire qu'ils vont dans les snowparks pour regarder ceux qui pratiquent. Soulignons que l'intérêt pour le côté convivial de la pratique (l'ambiance «cool » et le fait de se retrouver entre amis) croît au fur et à mesure que l'expertise augmente.

Les raisons pour lesquelles les pratiquants se rendent dans les snowparks varient aussi en fonction de l'âge. La principale différence concerne l'importance, chez les enfants (10-14 ans), de la dimension ludique de la pratique. Ainsi 55,7\% d'entre eux se rendent dans les snowpark «parce que c'est plus marrant que sur les pistes» et 66,4\% «pour avoir des sensations », contre respectivement $34,5 \%$ et $51,2 \%$ des $18-25$ ans. Soulignons qu'à niveau de pratique égal, la relation entre l'âge et les motivations exprimées ne perdure que chez les débutants. Il semblerait y avoir un processus d'acculturation ${ }^{7}$ ou d'imposition d'une certaine réalité de la pratique conduisant à une normalisation des motivations selon les niveaux.

Les pratiquants de surf des neiges et les skieurs ne diffèrent pas selon leurs motivations ; il en est de même en ce qui concerne les hommes et les femmes.

\section{Insérer ici figure 7}

\footnotetext{
${ }^{7}$ Mucchielli et Mucchielli (1969) définissent l'acculturation comme le «processus par lequel un individu apprend les modes de comportements, les modèles et les normes d'un groupe de façon à être accepté dans ce groupe et à y participer sans conflit ».
} 


\section{Discussion et conclusion}

Les pratiquants qui fréquentent les snowparks sont des hommes dans neuf cas sur dix, et des jeunes de moins de 18 ans dans cinq cas sur dix. Ils se rendent essentiellement en snowpark pour s'y adonner à des sauts, de manière souvent assidue, voire exclusive de toute autre modalité de pratique des sports d'hiver. Sans surprise, les pratiquants de niveau expert et confirmé sont essentiellement motivés par la pratique du freestyle, mais aussi par l'ambiance spécifique cultivée au sein des snowparks, alors que les débutants entendent surtout découvrir de nouvelles façons de pratiquer les sports d'hiver (plus ludiques, plus grisantes), et plus encore observer les autres pratiquants, ce qui renforce les interactions sociales propres à ces lieux.

Si les skieurs sont plus nombreux que les pratiquants de surf des neiges en snowpark, il convient néanmoins de réaliser que ces derniers sont proportionnellement deux fois plus nombreux dans ces espaces que sur le reste des domaines skiables. En d'autres termes, la pratique du surf des neiges, en déclin depuis le milieu des années 2000, est le fait d'une population vieillissante qui se concentre dans les snowparks, lesquels deviennent de fait une sorte de « réserve » pour les pratiquants de surf des neiges.

D'un niveau globalement moyen, la majorité des personnes pratiquant en snowpark n'hésitent pas à s'affronter à des modules de difficuté importante, potentiellement dangereux au regard de leur bagage technique et de leur expérience. Ce trait est renforcé pour les skieurs, bien plus nombreux que les pratiquants de surf des neiges à emprunter des modules de niveau très difficile (balisage noir).

A l'instar de ce qu'ont précédemment mis en exergue Anderson (1999) et Thorpe (2004, 2012) à propos de la pratique du surf des neiges, la domination masculine est un trait caractéristique de la fréquentation des snowparks. Au-delà du simple fait de fréquenter, ou non, ces espaces, la manière de le faire est aussi fortement influencée par le sexe des usagers.

$\mathrm{Au}$ sein des stations de sports d'hiver, les snowparks constituent des terrains de jeux singuliers. Au-delà de leur morphologie et du potentiel acrobatique qu'ils recèlent, la plupart des snowparks constituent aussi des lieux de vie à part entière, marqués par une organisation 
sociale et culturelle qui les situe en marge des normes traditionnelles des stations. Plus ludiques, plus « excitants », ils constituent des espaces aux usages multiples, qui vont bien audelà des seules évolutions acrobatiques: l'intensité des interactions sociales, l'ambiance spécifique et la recherche de convivalité les caractérisent également. Ces dimensions extrasportives sont d'ailleurs suffisamment structurantes pour entraîner la fréquentation «passive » des snowparks par une proportion importante de visiteurs.

En dépit de leur teneur essentiellement descriptive, les résultats présentés fournissent un éclairage relatif aux diverses catégories de personnes fréquentant les snowparks. La méthodologie employée, que ce soit au niveau de la réalisation du questionnaire ou de la construction de l'échantillon, nous permet d'affirmer que nos résultats, bien qu'issus d'une population mère inconnue, ont un sens et une portée réels. Ils sont représentatifs d'une population aux contours ignorés a priori (Martin, 2005). Globalement, bien que ces usagers se distinguent des autres clients des stations par leur jeunesse et une très nette sousreprésentation féminine, il s'avère que les snowparks ne sont pas « réservés » à une frange de skieurs experts, revendiquant leur appartenance à une culture sportive singulière (rappelons ici l'hétérogénéité des niveaux de pratique et des modalités de fréquentation).

Les principales conclusions de cet article sont susceptibles d'éclairer certaines décisions en matière d'aménagement des domaines skiables et de communication autour des espaces nouvelles glisses. En particulier, au regard de l'enjeu considérable que représentent désormais les snowparks des stations en termes d'attractivité commerciale, la très faible fréquentation féminine de ces lieux est susceptible de constituer un frein pour le développement de certaines sociétés d'exploitation des remontées mécaniques. De même, il semble important, sur le volet sécuritaire, d'œuvrer à une meilleure adéquation entre le niveau technique des pratiquants et les modules de saut qu'ils fréquentent, afin d'envisager une baisse de l'accidentalité constatée dans les snowparks, particulièrement élevée à ce jour (Reynier et al., 2014).

\section{Références}

Anderson, K. (1999). Snowboarding. The construction of gender in an emerging sport. Journal of Sport \& Social Issues, 23 (1), 55-79. DOI: 10.1177/0193723599231005 Apilli, E. (2007). L'ancrage institutionnel et territorial spécifique d'une contre-culture 
assagie : le snowboard. In P. Bourdeau (Ed.) Les sports d'hiver en mutation. Crise ou révolution géoculturelle? (pp. 67-74). Paris : Lavoisier.

Berthier, F., \& Berthier, N. (1971). Le sondage d'opinion. Paris : Bordas.

Brooks, M.A., Evans, M.D., \& Rivara, F.P. (2010). Evaluation of skiing and snowboarding injuries sustained in terrain parks versus traditional slopes. Injury Prevention, 16 (2), 119-122. DOI : $\underline{10.1136 / i p .2009 .022608}$

Coates, E., Clayton, B., \& Humberstone, B. (2010). A battle for control: exchanges of power in the subculture of snowboarding ». Sport in Society, 13 (7/8), 1082-1101. DOI : $10.1080 / 17430431003779999$

Curtet, J. (2007). L'offre d'espaces nouvelles glisses en France: vers un bilan critique. In P. Bourdeau (Ed.) Les sports d'hiver en mutation. Crise ou révolution géoculturelle? (pp. 4756). Paris : Lavoisier.

Curtet, J. (2011). Snowparks ou espaces assimilés. Etat des lieux en France. E-Cahiers de l'ENSM, 2, 50-53.

Drouet, Y., Kemo Keimbou, D.C. (2005). Comment devient-on Freerider ? Une approche socio-anthropologique. Loisir \& Société, 28 (1), 67-88.

Dupuy, N. (2007). Le ski alpin entre crise, ruptures technologiques et renouveau. In P. Bourdeau (Ed.) Les sports d'hiver en mutation. Crise ou révolution géoculturelle? (pp. $57-$ 64). Paris : Lavoisier.

Edensor, T., \& Richards S. (2007). Snowboarders vs skiers: Contested choreographies of the slopes. Leisure Studies, 26 (1), 97-114. DOI : 10.1080/02614360500372224

Feuillie, B. (2011). Incidence de risque de blessure lors de la pratique des sports d'hiver dans les snowparks (thèse de doctorat en médecine non publiée). Université Grenoble 1, Grenoble. Goulet, C., Hagel, B., Hamel, D., \& Légaré, G. (2007). Risk factors associated with serious ski patrol reported injuries sustained by skiers and snowboarders in snow-parks and on other slopes. Can J Public Health, 98 (5), 402-406.

Guibert, C. (2006). Les usages politiques du snowboard dans les stations de montagne françaises : entre intérêt économique et dédain culturel. Revue européenne de management du sport, 15, 31-44.

Heino R. (2000). New Sports: What is so punk about snowboarding? Journal of Sport \& Social Issues, 24 (2), 176-191. DOI: 10.1177/0193723500242005

Humphreys, D. (1997). Shredheads Go Mainstream ? Snowboarding and Alternative Youth. International Review for the Sociology of Sport, 32, 147-160. DOI : 10.1177/101269097032002003 
Laporte, J.D. (2011). L'accidentologie dans les snowparks. e-Cahiers de l'ENSM, 2, 95-98.

Médecins de Montagne (2013). Accidentologie des sports d'hiver. Dossier de presse.

Lamprecht, M., Fischer, A. \& Stamm, H.P. (2015a). Sport Suisse 2014: Les sports en chiffres.

Macolin : Office fédéral du sport OFSPO.

Lamprecht, M., Fischer, A., Wiegand, D., \& Stamm, H.P. (2015b). Sport Suisse 2014 : rapport sur les enfants et les adolescents. Macolin : Office fédéral du sport OFSPO.

Martin, O. (2005). L'enquête et ses méthodes. L'analyse de données quantitative. Paris: Armand Colin.

Mucchielli, A., \& Mucchielli, R. (1969) Lexique des sciences sociales. Paris : Entreprise Moderne d'Édition, Éditions Sociales Françaises,

Pabion-Mouriès, J., Reynier, V., \& Soulé, B. (2014) Bodily engagement of snowpark freestylers: A study in three french winter sports resorts. International Review for the Sociology of Sport 51 (5), 581-595. DOI : 10.1177/1012690214545901

Reynier, V., \& Chantelat, P. (2005). Les comportements territoriaux des pratiquants des stations de sports d'hiver. Loisir \& société, 28 (1), 49-66.

Reynier, V., Soulé B., \& Pabion-Mouriès, J. (2014). Prévention des risques en snowpark: observation des usages, analyse des formes d'engagement corporel, étude des représentations et formulation de préconisations. Paris : Fondation MAIF.

Rinehart, R.E., \& Sydnor, S. (Ed.) (2003). To the Extreme: Alternative Sports, Inside and Out. Albany : State University of New York Press.

Ruedl, G., Kopp, M., Sommersacher, R., Woldrich, T., \& Burtscher, M. (2013). Factors associated with injuries occured on slope intersections and in snowparks compared to onslope injuries. Accident Analysis and Prevention, 50, 1221-1225. DOI : 10.1016/j.aap.2012.09.019

Russell, K., Meeuwisse, W., Nettel-Aguirre, A., Emery, C.A., Wishart, J., Romanow, N.T., Rowe, B.H., Goulet, C., Hagel, B.E. (2013). Characteristics of injuries sustained by snowboarders in a terrain park. Clin. J. Sport. Med., 23 (3), 172-177. DOI : 10.1136/bjsports$\underline{2012-091912}$

Soulé, B., Reynier, V., \& Pabion-Mouriès, J. (2016). Snow Park evolution in France from 1990 to 2010: a plurality of compromises and gradual rationalisation behind a major territorial innovation. Society \& Leisure, 39 (1), 104-121. DOI : 10.1080/07053436.2016.1151225

Thorpe, H. (2004). Embodied boarders: snowboarding, status and style. Waikato Journal of Education, 10, 181-201.

Thorpe, H. (2011). Snowboarding bodies in theory and practice. London : Palgrave 
Macmillan. DOI : 10.1057/9780230305571

Thorpe, H. (2012). Transnational mobilities in snowboarding culture: Travel, tourism and lifestyle migration. Mobilities, 7 (2), 317-345. DOI : 10.1080/17450101.2012.654999

Torjussen, J., \& Bahr, R. (2006). Injuries among elite snowboarders (FIS Snowboard World Cup). Br J Sports Med, 40 (3), 230-234. DOI : 10.1136/bjsm.2005.021329

Vermeir, K., \& Reynier, V. (2008). Le risque sur les domaines skiables alpins. Relations entre sport pratiqué et représentations sociales des pratiquants. Science \& Motricité, 64, 69-81.

Wheaton, B., \& Beal, B. (2003). Keeping it real. Subcultural media and the discourses of Authenticity in alternative sports. International Review for the Sociology of Sport, 38 (2), 155-176. DOI : $10.1177 / 1012690203038002002$

Woermann, N. (2012). On the slope is on the screen. Presumption, social media practices and scopic systems in the freeskiing subculture. American Behavioral Scientist, 56, 618-640. DOI: $10.1177 / 0002764211429363$ 